la comparaison des écarts de poids des veaux charolais ("N") et limousins exploité en zone de race pure d'une part et de ceux des veaux croisés issus de taureaux charolais (" $\mathbf{M}$ ") et limousins utilisés sur le même cheptel femelle (sud-ouest de la France) d'autre part, permet d'estimer la différence de valeur génétique (M-N) entre ces deux types de Charolais.

Ces différences concordent aussi bien avec d'autres expériences françaises réalisées sur bovins normaux et culards, qu'avec les variations, constatées dans divers pays américains selon l'origine des taureaux charolais, de la supériorité des descendants de mâles charolais sur celles des taureaux Hereford ou Aberdeen-Angus.

Tous ces résultats traduisent l'évolution du potentiel de croissance sous l'effet de la sélection naturelle (conditions d'élevage) et artificielle (recherche de la musculature). L'écart minimun de poids à la naissance (20 p. IOO) paraissant supérieur à celui entre les poids adultes des types extrêmes, cela tendrait à indiquer qu'il y a modification de la forme de la courbe de croissance avec l'augmentation de la musculature.

\title{
L'INTERFÉRENCE DU CARACTÈRE “CULARD » ETT DE LA SÉLECTION BASÉE SUR LA CONFORMATION, DANS LA RACE BOVINE DE MOYENNE ET HAUTE-BELGIQUE
}

\author{
R. Hanset. -- Chaire de Génétique, Faculté de Médecine vétérinaive, Université de Liège, \\ Cureghem-Bruxelles (Belgique).
}

En race de Moyenne et Haute-Belgique, se poursuit une sélection en faveur d'une conformation caractérisée par l'arrière-main et la ligne du dessus fortement musclées, la croupe inclinée, les hanches effacées, la côte arrondie. L'auteur se livre à un bilan de cette sélection, du point de vue du caractère culard, dans deux provinces représentatives. De I958 à I970, la fréquence du caractère culard, dans la descendance de l'insémination artificielle, est passée de 2,28 p. Ioo à 9,I p. Ioo dans une province, de I,43 p. Ioo à $7,13 \mathrm{p}$. I oo dans la seconde. La fréquence des césariennes est de l'ordre de i i p. Ioo dans les deux provinces. Si on caractérise chaque taureau IA par la proportion de ses descendants qui présentent le caractère culard, on s'aperçoit qu'il existe une grande hétérogénéité entre taureaux. Un déterminisme monogénique pour le caractère culard est compatible avec les données d'observation à la condition d'admettre l'intervention de gènes modificateurs (au moins une paire) à grande amplitude d'action. L'évolution de la conformation, dans la descendance normale des mêmes taureaux IA est étudiée parallèlement à l'évolution de la fréquence du caractère culard. La relation entre ces deux événements est faible. Dans la population considérée, le résultat de la sélection basée sur la conformation aurait été d'accroître la fréquence du gène culard, d'où la fréquence du caractère, sans pour autant avoir amélioré d'une façon sensible le développement moyen de la musculature des animaux non-culards.

\section{Essai DE CARActÉRISATION BIOChIMIQUE DU MUSCLE DE BOVINS CULARDS (1)}

\author{
M. Ansay. - Chaire de Génétique, Faculté de Médecine vétérinaire, Université de Liège, \\ Cureghem-Bruxelles. (Belgique).
}

La teneur en ADNP du muscle demi-tendineux chez des animaux culards et normaux a été examinée depuis le troisième mois de la vie fœetale jusqu'à l'âge adulte. La concentration en ADNP reste élevée (20 à $25 \mathrm{mg}$ d'ADNP) du troisième au cinquième mois environ de la: vie fotale, puis diminue progressivement pour passer à Io $\mathrm{mg}$ environ à la naissance, à $2 \mathrm{mg}$ à l'âge de 15 mois. Pendant toute la période envisagée, les animaux culards ont une concentration équivalente en ADNP.

(1) Travail exécuté sous les auspices de l'Institut pour l'encouragement de la Recherche scientifique dans l'Industrie et l'Agriculture. 
La comparaison des différentes fractions azotées dans le muscle peaucier d'animaux de I 8 mois révèle que la concentration de l'azote sarcoplasmique est plus élevée chez l'animal culard $(27,1$ I p. I 00 contre 24,55 p. Ioo de l'azote soluble). L'azote du stroma représente I 3,85 p. Ioo de l'azote total chez les animaux normaux, 8,79 p. Ioo chez les culards. La concentration en myoglobine de ce muscle apparaît équivalente chez les 2 types d'animaux. A l'âge moyen de 42 jours (culards) et de 62 jours (normaux) les rapports GAPDH/EC, GAPDH/HOAD, LDH/EC sont semblables dans le muscle semi-tendineux ou long vaste. Seul le rapport phosphorylase/HK apparaît abaissé chez les culards. Tous ces rapports sont augmentés chez l'animal adulte normal et diminués dans le cceur. On n'a pas trouvé de différences quant au pourcentage de sujets hétérozygotes, pour les loci Mor-I et PMGI, chez les animaux normaux ou culards.

\section{CONSÉQUENCES DÉ L'HYPERTROPHIE MUSCULAIRE HÉRÉDITAIRE DES BOVINS SUR LA MUSCULATURE \\ I. - IMPORTANCE REIATIVE DES DIFFÉRENTES RÉGIONS}

R. Boccard et B.-L. Dumont. - Station de Recherches sur la Viande, C. R. Z. V., I. N. R. A., Theix, 63 - Saint-Genès-Champanelle (France). - Laboratoire de Recherches sur la Viande C. N.R. Z., I. N. R. A., 78-Jouy-en-Josas (France).

Les résultats de la dissection complète de la musculature des demi-carcasses de 9 taurillons hypertrophiés et de $\mathbf{I} 7$ taurillons normaux de race charolaise de Io à 20 mois ont été traités pour comparer la part relative des différentes régions musculaires. La comparaison a été effectuée après calcul par régression, pour chaque région, du poids qu'elle représentait dans une masse de $\mathrm{I}_{4} \mathrm{O} \mathrm{kg}$ de muscle dans une demi-carcasse. Les différents muscles ont été regroupés en onze régions dont l'importance relative a été appréciée par le rapport $\frac{\mathrm{H}-\mathrm{N}}{\mathrm{N}} \times$ roo où $\mathrm{H}$ est le poids de la région du type hypertrophié et $\mathrm{N}$ le poids de celle du type normal. Les valeurs obtenues furent pour chaque région : cervicale $-6,3 \mathrm{I}$; antibrachiale $+0,16$; brachiale $+5,02$; scapulaire $-5,72$; rachidienne $-8,46$; thoracique +6.77 ; diaphragmatique - I8,05; abdominale $-5,84$; fessière $-4,44$; crurale $+9,92$ et jambière $-6,44 \mathrm{p}$. Ioo.

Il apparaît donc qu'à poids de muscle constant l'hypertrophie affecte uniquement les régions crurale, thoracique et brachiale et que les autres régions sont retardées dans leur développement à des degrés divers.

Les modifications constatées dans l'importance relative des masses musculaires des différentes régions constituent une exception à la loi d'harmonie anatomique. La différence de répartition entre régions explique, en partie, les différences d'aspect et de conformation qui séparent les deux types d'animaux (finesse de l'encolure, ventre levretté, membre postérieur dit " cul de poulain ") et que l'on peut apprécier sur une base quantitative d'après la valeur du rapport du poids des muscles de la cuisse au poids des muscles du cou $(2,27$ pour les hypertrophiés contre 1,94 pour les normaux) ou encore à celui des muscles de la région abdominale inférieure $\left(3,98\right.$ pour les hypertrophiés contre $3,3^{6}$ pour les normaux).

\section{CONSÉQUENCES DE L'HYPFRTROPHIE MUSCULAIRE HÉRÉDITAIRE DES BOVINS SUR LA MUSCUL,ATURE}

\section{II. - IMPORTANCE RELATIVE DES DIFFÉRENTS MUSCLES}

B.-L. Dumont et R. Boccard. - Laboratoive de Recherches sur la Viande, C. N.R. Z., I. N. R. A., 78 - Jouy-en-Josas (France). - Station de Recherches sur la Viande, C. R. Z. V., I. N.R. A., Theix, 63-Saint-Genès-Champanelle (France).

On a comparé, après calcul par régression des poids qu'ils représenteraient dans une masse de $140 \mathrm{~kg}$ de muscle par demi-carcasse, le poids des principaux muscles de taurillons charolais de Io à 20 mois, de type normal $(n=$ I 7$)$ et de type hypertrophié dit " culard " $(n=9)$. La 